\title{
Klima und Lufthygiene der Region Biel - Gedanken und erste Resultate aus einer interdisziplinären Studie
}

\section{Einleitung}

Nach der Zürcher Definition (IrTEN 1982) untersucht die Geographie Natur und Gesellschaft mit dem Ziel, räumliche Systeme und Prozesse zu erklären. Das Gebilde einer Stadt darf ohne Zweifel als eines der komplexesten dynamisch-räumlichen Systeme bezeichnet werden. Dies ist mit ein Grund, daß die Stadtklimatologie innerhalb der physischen Geographie weltweit zu einem integrierenden Forschungszweig geworden ist (BACH 1970, ERIKSEN 1975, OKE 1978, MATHYs et al. 1980, weISCHET 1980, TAPPER et al. 1981). Beschränkte man sich früher auf eine Messung und Beschreibung der Klimaelemente oder bestenfalls auf eine empirisch-statistische Analyse des Datenmaterials in ausgewählten Einzelstädten, so konnte in den letzten zehn Jahren eindeutig eine Trendwende in Richtung allgemeine Prozeßanalyse, Prozeßverständnis und «Modeling» beobachtet werden (OKE 1982). Was auch diesen modernen Arbeiten häufig abgeht, ist die direkte Umsetzung der stadtklimatologischen Erkenntnisse in die Raumplanung und in politische Entscheidungsprozesse (KLIMA UND PLANUNG 79). Der Geograph ist auf Grund seiner Ausbildung prädestiniert, aktiv in diesen angewandten und interdisziplinär ausgerichteten Aufgabenkreis einzusteigen. Der folgende Beitrag beschreibt eine Studie, die zur Zeit in der Stadtregion Biel durchgeführt wird. Neben der Untersuchung von Stadtklima und Lufthygiene wird eine dauernde Umsetzung der Ergebnisse in die laufenden raumplanerischen Entscheidungsprozesse angestrebt!

\section{Probleme und Ziele}

Im Jahre 1979 gelangte der Regionalplanungsverein Biel-Seeland mit der Bitte an uns, die herrschenden klimatologisch-lufthygienischen Verhältnisse in der Region Biel näher zu untersuchen, um bessere Entscheidungsgrundlagen für die anstehenden Planungsentscheide (u.a. Autobahnlinienführung N5/ T6, Planung neuer Siedlungs- und Industriezonen, Erschließung von Naherholungsgebieten, städtisches Energiekonzept) zu erhalten. Die Studie sollte auch den biologischen Folgewirkungen von Klima und Lufthygiene Rechnung tragen. Da nur sehr beschränkt Finanzen zur Verfügung standen, mußten
A. die Arbeiten auf planungsrelevante Fragen beschränkt und

B. Institutionen mit einer vorhandenen personellen und materiellen Infrastruktur (Universitätsinstitute, Verwaltungsstellen) in die Studie eingeschaltet werden.

Die Region Biel weist als Teil der tieferen Mittellandsenke im Winter eine Häufung von austauscharmen Stagnationslagen mit Inversionen, Schwachwinden und Nebelmeeren auf, was durch eine Vorstudie bestätigt wurde (EGLI und WANNER 1980). Eine erste Diskussion zwischen Raumplanern und verschiedenen Naturwissenschaftern ergab, daß sich die klimatologischen Untersuchungen auf die Erfassung des Ausbreitungsklimas (mit Schwerpunkt auf dem Winterhalbjahr) beschränken mußten. Aus diesem Grund wurde zuerst ein einfaches Wirkungsgefüge aufgestellt, das die für die Ausbreitung von Luftfremdstoffen wichtigen Einflußgrößen abgrenzt (Fig. 1): Im zentralen Bereich der Figur erkennen wir jene Mechanismen, welche entscheidend zur Verdünnung oder Umwandlung einer Anfangskonzentration $x_{i}$ zur Endkonzentration $x_{f}$ beitragen. Die doppelt umrandeten Einflußgrößen zeigen, daß die Untersuchungen auf die für den Transport und die turbulente Diffusion entscheidenden Einflußgrößen Horizontalwindfeld, Vertikaltemperaturschichtung und Nebel einerseits (meteorologische Parameter) und Topographie, Bodenrauhigkeit und Bodenbedeckung andererseits $(\mathrm{Ge}$ ländeparameter) zu konzentrieren sind. Die für diese Arbeiten zuständige Arbeitsgruppe legte die folgenden Arbeitsziele fest:

A. Die Wirkungskette Emission (als quellenseitige Anfangsbelastung) - Transmission (Ausbreitungsklima) - Immission (räumliches Muster der Endbelastung) soll mit Schwerpunkt auf Fallstudien (v. a. bei durchlüftungsarmen Wetterlagen im Winterhalbjahr) studiert werden.

B. Die Ergebnisse sind mit Untersuchungen der Folgewirkungen zu korrelieren; die Resultate sollen im Rahmen einer Arbeitsgruppe direkt in den raumplanerischen Entschiedungsproze $B$ einfließen.

Heinz Wanner, Pierre Berlincourt und Ralph Rickli

Geographisches Institut

Hallerstraße 12, 3012 Bern 


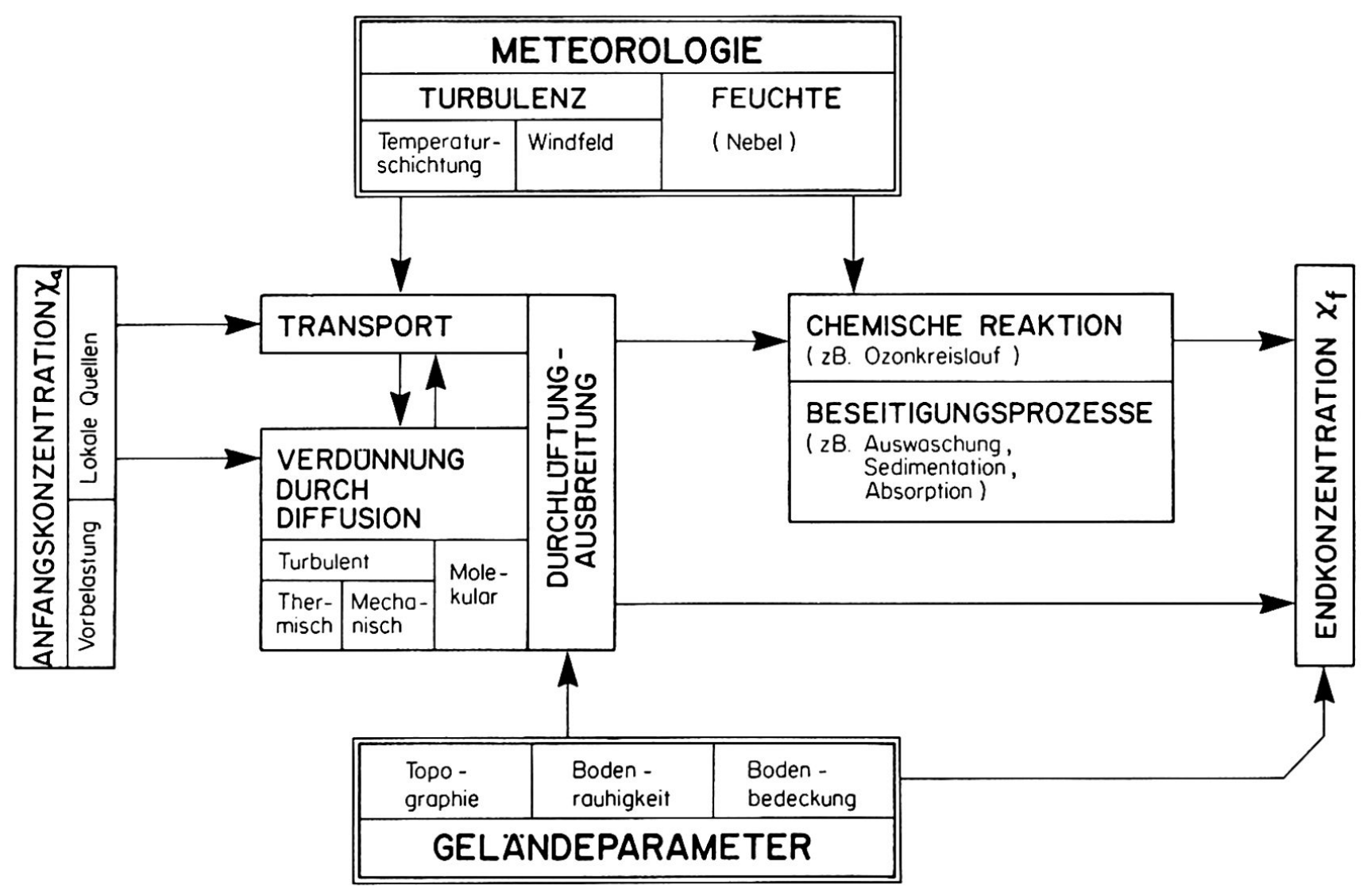

Fig. 1 Vereinfachtes Wirkungsgefüge zur Ausbreitung von Luftfremdstoffen

\section{Arbeitskonzept}

Die Zielsetzungen wurden in das auf Figur 2 gezeigte Arbeitskonzept umgesetzt. Folgende Stichworte geben einen Überblick über Arbeiten und beteiligte Institutionen (Abkürzungen siehe unten):

- Emission: Entwurf eines raumzeitlich fein aufgelösten Emissionskatasters (Hektarraster) auf der Basis der Ermittentengruppen Industrie, Gewerbe, Hausbrand und Verkehr (P. BERLINCOURT, GIUB; H. MATHYS, KIGA).

- Transmission/Klima: Messung sowie theoretische Abschätzung der in Figur 1 doppelt umrandeten Einflußbereiche Meteorologie und Geländeparameter (R. RICKLI und H. WANNER, GIUB).

- Klima/Modelle: Modellierung im Windkanal (J. HERTIG, IENER) und anhand eines einfachen, empirisch-statistischen Ausbreitungsmodells ( $\mathrm{H}$. WANNER, GIUB).
- Immission: Auswertung der Langzeitmessungen des Bieler Stadtnetzes $\left(\mathrm{SO}_{2}\right.$, Staub: P. BERLINCOURT, GIUB) sowie Durchführung episodischer Kurzzeitmessungen $\left(\mathrm{SO}_{2}, \mathrm{NO}_{\mathrm{X}}\right.$ : $\mathrm{P}$. BERLINCOURT und $\mathrm{R}$. RICKLI, GIUB, sowie h. MATHYS, KIGA).

- Folgewirkungen: Statistische Erfassung akuter Atemwegserkrankungen bei Kleinkindern im Winterhalbjahr (H. MARTY, Bezirksspital Zweisimmen; R. ZURBRÜGG, Kinderspital Wildermeth, Biel; J. KLINGLER, Kinderarzt, Biel) sowie Untersuchungen an Flechten (K. AMMANN, Botanisches Institut Bern).

- Raumplanerische Maßnahmen: Umsetzung der Ergebnisse in die Raumplanung (Planungsbüro BERZ + DROux Bern im Auftrag des Regionalplanungsverbandes Biel-Seeland).

(GIUB: Geographisches Institut der Universität Bern; KIGA: Kantonales Amt für Industrie und Gewerbe, Bern;

IENER: Institut d'économie et aménagements énergétiques, EPF Lausanne)

Bei der nun folgenden Darstellung erster Resultate sei das Ausbreitungsklima der Region Biel in den Vordergrund gestellt. 


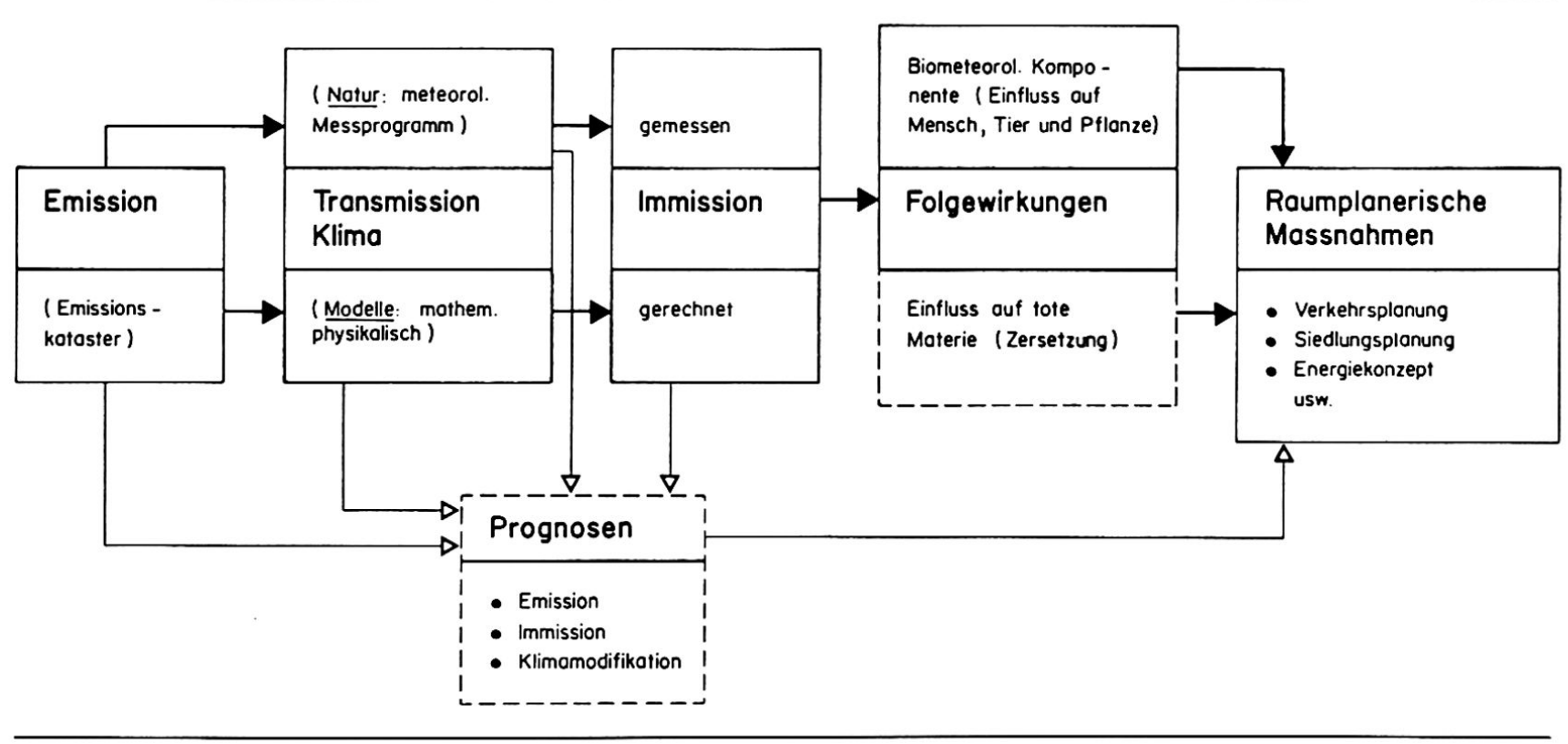

Fig. 2 Arbeitskonzept “Klima und Lufthygiene Biel» (gestrichelt: möglicher Weiterausbau des Projektes)

\section{Zum Ausbreitungsklima der Region Biel}

$\mathrm{Zu}$ Beginn wird eine theoretische Grobabschätzung jener Einflüsse vorgenommen, welche die Geländeparameter (als relativ invariable Größen) auf die für die Luftfremdstoffausbreitung maßgeblichen Klimaelemente ausüben. Figur 3 vermittelt einen Überblick über diese Geländeparameter sowie über das Windfeld der Untersuchungsregion.

\subsection{Topographie}

Die südlichste Jurakette im NW und die beiden Molassehügel im E (Längholz, Büttenberg) bewirken zunächst eine markante aerodynamische Beeinflussung der synoptischen Windsysteme (Kanalisierung SW - NE). Im weiteren induzieren sie als erhöhte Wärmequellen (tagsüber) und als Orte der Kaltluftproduktion und des Kaltluftabflusses (nachts) sehr wichtige thermische Windsysteme in Form von Hang-, Berg- und Talwinden. Die zwischen den Hügeln eingebetteten Mulden erweisen sich demgegenüber als ausgesprochene Kaltluftsammelbecken.

\subsection{Bodenrauhigkeit}

Die Rauhigkeitslänge $z_{0}$ ist für die Abschätzung der dynamischen Turbulenz (v. a. Vertikalwindprofil) von fundamentaler Bedeutung. Eine erste Schätzung ergibt für die Region Biel die folgenden Rauhigkeitslängen (LETTAU 1969, OKE 1978, SPERA und RICHARDS 1978; siehe Fig. 3):

- Wasser (weiß): $\quad 0,1-10,0 \cdot 10^{-5} \mathrm{~m}$

- Äcker, Felder, Gras (weiß): 0,002-0,1 m

- Wald (grau): $\quad 1,0-2,0 \mathrm{~m}$

- Stadt (Backsteinmuster): $\quad 0,07-1,5 \mathrm{~m}$
Verallgemeinert kann gesagt werden, daß mehr als $2 / 3$ des Untersuchungsgebietes als "rauhe Oberfläche» angesprochen werden darf, eine Tatsache, die zusammen mit der oben erwähnten Beckenstruktur bereits auf Austauschprobleme hindeutet!

\subsection{Bodenbedeckung}

Bodenbedeckung und Solarkonstante stellen die wichtigsten (relativ) stabilen Steuerungsgrößen des Strahlungs- und Energiehaushaltes und somit der Auslösung thermodynamischer Prozesse innerhalb der «Planetary Boundary Layer» (PBL) dar. Im Falle Biels dämpft der See auf Grund seiner hohen Wärmekapazität die Temperaturextreme sowohl im Tages- als auch im Jahresgang - ein Effekt, der vermindert auch den Waldflächen eigen ist. Zusammen mit Acker- und Wiesenflächen bilden Wald und See zudem wichtige Ausgangsreservoire des Flusses latenter Wärme. Nicht so die Stadt: Gegenüber dem Umland weist sie sich infolge ihrer relativen Trockenheit, ihres hohen thermischen Speichervermögens (OKE 1982) und ihrer Strahlungsgeometrie ("sky view factor», OKE 1981) vor allem während der Nacht durch einen massiv erhöhten Input sensibler Wärme an die bodennahe Atmosphäre aus. Zusammen mit den orographischen Effekten dürfte sie als «trocken-rauhe Wärmeinsel» einen entscheidenden Einfluß auf die Auslösung turbulenter Prozesse innerhalb der PBL ausüben!

Der Blick sei nun auf die für die Durchlüftung entscheidenden Klimaelemente Wind- und Temperaturfeld gerichtet. 


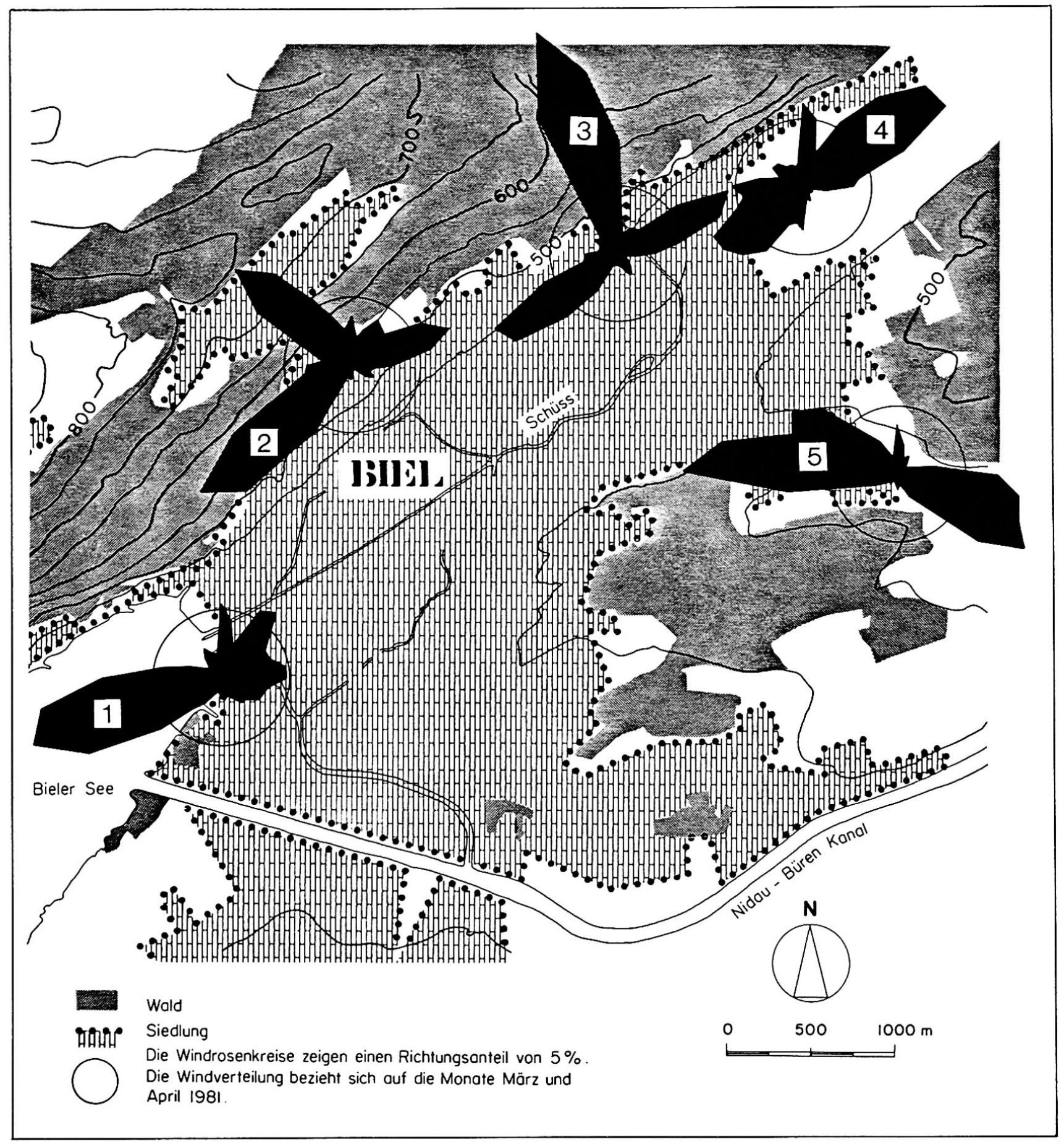

Fig. 3 Räumliche Verteilung der Geländeparameter Topographie und Bodenbedeckung sowie Darstellung wichtiger Windrosen

\subsection{Windfeld}

Die Windrosen der Monate März und April 1981 (Fig. 3) zeigen die oben erwähnten Kanalisierungseffekte. Bei der Station Vogelsang (Jurahang, Nr. 2) zeigt sich ein deutlicher Hangwindeinfluß, welcher bei der Station Bözingen (Nr.3) durch den markanten Kaltluftabfluß aus dem südlichsten Juratal («Taubenlöchler») verstärkt wird. Die Stationen Strandbad (Nr. 1) und Bözingenmoos (Nr.4) liefern anhand der überwiegenden «Landwindsektoren» (Flurwinde?) eindeu- tig den Nachweis für den Stadteinfluß (Thermik, Reibungskonvergenz), wobei im Falle der Station Strandbad zusätzlich ein Seewindeinfluß mitspielen dürfte.

\subsection{Temperaturfeld}

Figur 4 gibt anhand des 10. Dezember 1980 einen Überblick über das bodennahe Lufttemperaturfeld (inkl. verfügbare Windangaben) und den durch Sondierungen im Stadtzentrum ermittelten Tagesgang 

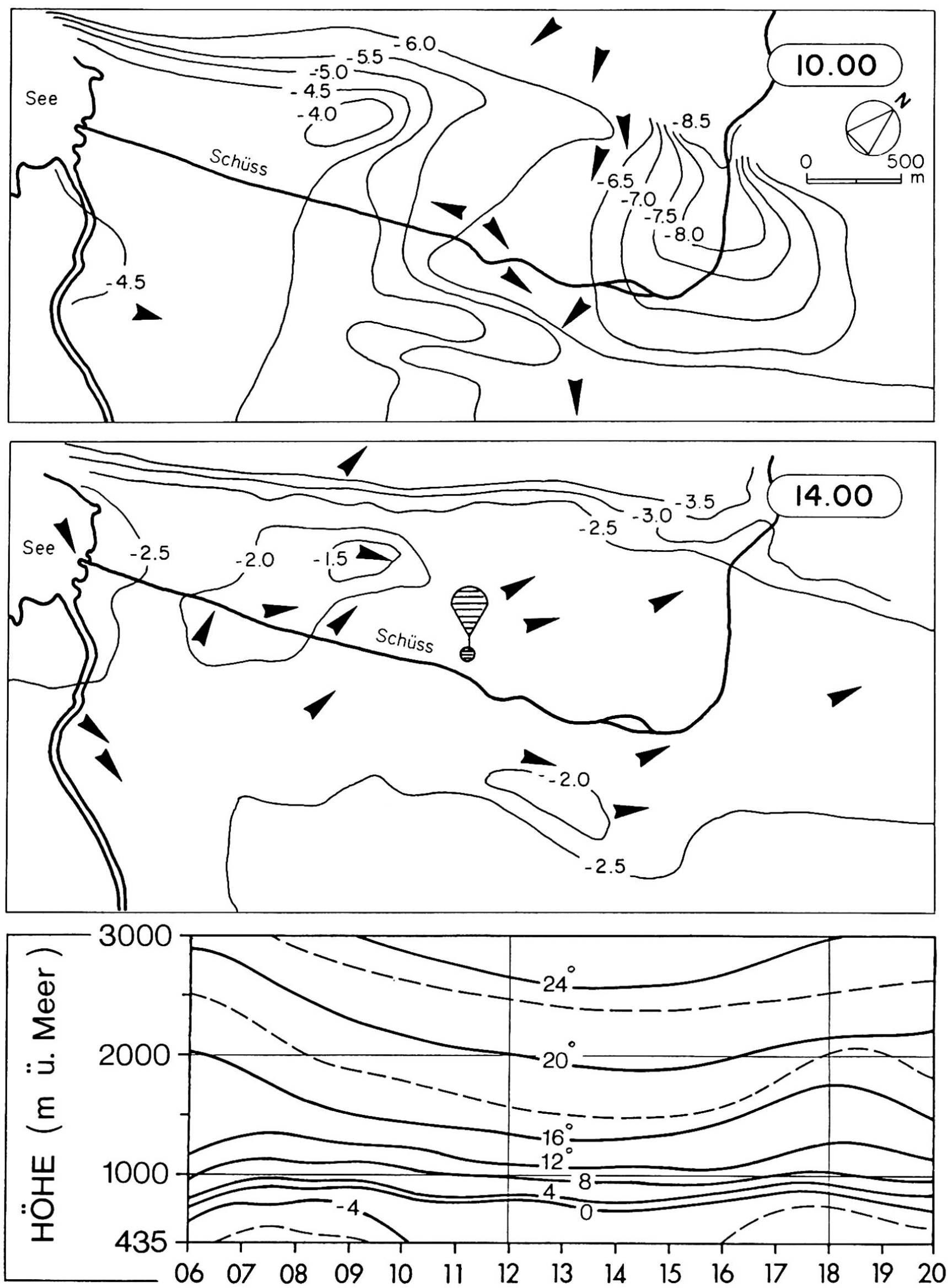

Fig. 4 Antizyklonale Nordlage vom 10. Dezember 1980: räumliche Konfiguration des Temperatur- und Windfeldes um 10.00 und 14.00 Uhr sowie Tagesverlauf der Vertikaltemperaturschichtung im Stadtzentrum (potentielle Temperaturen; Meßstandort: Schulhaus Rittermatte; siehe unterste Fig.) 
Austauschbegünstigte, nebelarme Zone oberhalb der Mischungsschicht

$\because:::$ Bereich der Mischungsschichtobergrenze (oft Höheninversion mit Hochnebel und schwachen Winden); starke Luftfremdstoffanreicherung tagsüber infolge Hangaufwind und "Sperreffekt"

Warme und nebelarme Zwischenzone im Bereich der Hangwindsysteme (teilweise austauschgefährdet)
Austauschgefährdete Talzone mit geringer bis mittlerer Bodennebelhäufigkeit

Austausch- und kaltluftgefährdete Zone mit großer Bodennebelhäufigkeit
Gradientwinde
- durch Gradient- und Corioliskraft be- stimmt

- keine thermische Beeinflussung

Höhenwinde

- oberhalb Mischungsschicht

- leichter Schubspannungseffekt (Topographie)

- keine unmittelbare thermische Beeinflussung (ohne Tagesgang)

Regionalwinde (100-200 m ü. Boden)

- im Bereich der unteren Drehungsschicht

- Schubspannung überwiegt Gradientund Corioliskraft (Grossrelief)

- nachweisbare thermische Beeinflussung (Tagesgang)

Lokalwinde ( $10 \mathrm{~m}$ ü. Boden)

- im Bereich der turbulenten Bodenschicht

- Schubspannung dominiert eindeutig über Gradient- und Corioliskraft (Klein. relief, Rauhigkeitselemente)

- starke thermische Beeinflussung (Bodenbedeckung als Leitgröße, Tagesgang klar sichtbar)
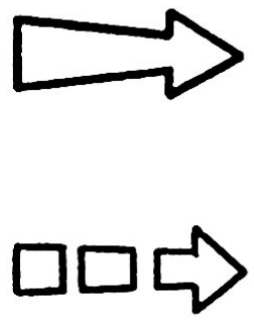

पर Berg- (B) und Talwinde ( $T$ ) in Hauptströmungsachsen

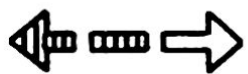

Berg- (B) und Talwinde ( $\mathrm{T}$ ) in Nebenströmungsachsen

Hangauf- (AF) und Hangabwinde (AB) sowie Windsysteme in kleinen Tälern und Kaltluftflüsse (KF)

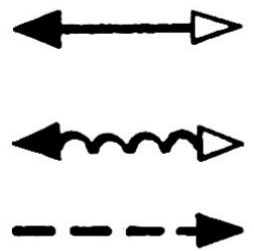

Land- $(L)$ und Seewinde (S)

Vermutete Flurwinde

Legende zu Fig. 5

der Schichtung der potentiellen Temperatur. Es handelt sich um eine der für die Studie wichtigen winterlichen Stagnationslagen (antizyklonal, Höhenströmung N). Um 10.00 Uhr lassen sich sowohl die Wärmeinsel des Altstadtkerns (sehr tiefer "sky view factor»!) als auch der Kaltluftabfluß aus dem Taubenloch deutlich feststellen. Wie in andern Städten gleichen sich die Temperaturgegensätze um 14.00 Uhr aus (stärkerer Schattenwurf und höheres thermisches
Speichervermögen im Stadtraum), und die Musterung des Windfeldes deutet auf Hang- und Seewindeffekte hin. Die Potentialtemperaturschichtung (unterste Fig.) als $\mathrm{Maß}$ der Vertikalstabilität zeigt eine durchgehend stabil geschichtete PBL mit einer besonders engen Scharung der Isothermen zwischen 600 und $1200 \mathrm{~m}$ über Meer. Dieser für die Turbulenz als "Sperrschicht» wirkende Layer weist infolge der starken Subsidenz und der vorhandenen Nebel- oder Dunst- 


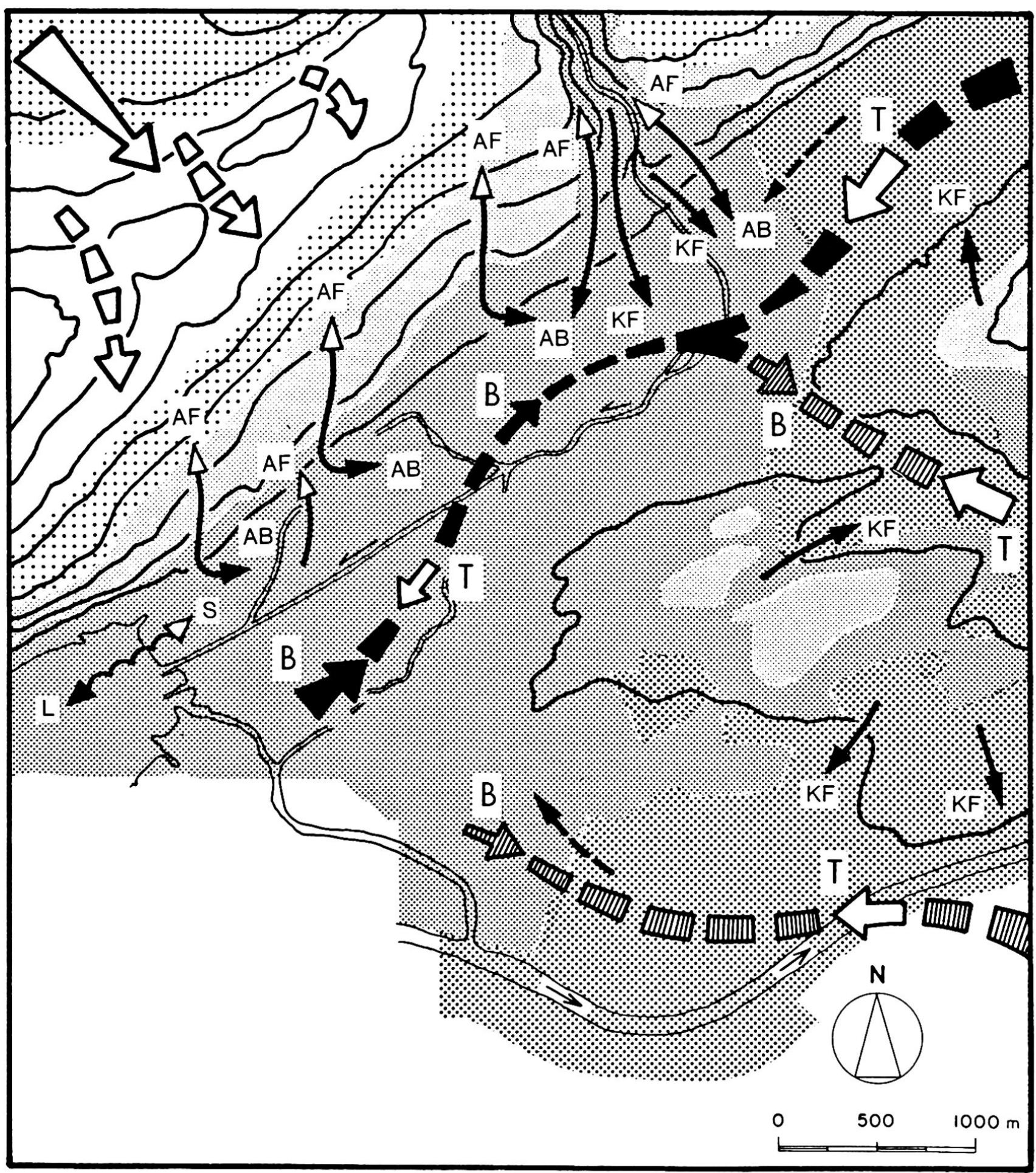

Fig. 5 Durchlüftungskarte für winterliche Hochdrucklagen mit nordwestlicher Höhenströmung. Die Pfeilbreiten richten sich nach der zu erwartenden Windgeschwindigkeit

schicht trotz der stadtinternen Aufheizung der unteren PBL überhaupt keine Tendenz zur Auflösung auf. Es kann vielmehr beobachtet werden, daß die auf Grund der durchdringenden und im Bereich der komplexen Stadtoberfläche absorbierten, kurzwelligen Strahlung dennoch in Gang gesetzten Jura-Hangaufwinde an der Untergrenze dieser stabilen Schicht gekappt werden.

\subsection{Durchlüftungskarte}

Infolge der großen lufthygienischen Bedeutung und Häufigkeit antizyklonaler, winterlicher Nordwest-, Nord- und Nordostlagen wurde versucht, eine Durchlüftungskarte einer derartigen Wetterlage zu entwerfen (Fig.5). Diese Karte gibt die für die Ausbreitung bedeutungsvollen Phänomene Wind, Temperatur- 
schichtung und "Schönwetternebel» auf einer einzigen Darstellung wieder (WANNER 1982). Neben den austauschgefährdeten Kaltluftsammelgebieten wird am Jurahang auch der sehr oft als "Sperrschicht» wirkende Bereich der oben erwähnten Inversionsuntergrenze (ungefähr identisch mit der Mischungsschichtobergrenze; FETT 1974) sehr deutlich sichtbar. Ein wichtiges Hauptziel der Bieler Arbeiten ist es nun, diese Durchlüftungsmechanismen mit den Immissionsmessungen und den Analyseergebnissen von Folgewirkungen in Beziehung zu bringen, um daraus allenfalls Planungsentscheide abzuleiten!

\section{Immissionen, Folgewirkungen und erste Auswirkungen auf Planungsentscheide}

\subsection{Die Immissionsgefährdung anhand von $\mathrm{SO}_{2}$-Messungen}

Zur Zeit liegen lediglich $\mathrm{SO}_{2}$ und Staub ausgewertet vor (AESCHBACHER und MELI 1977, BERLINCOURT 1981). Aus Platzgründen sei hier nur die $\mathrm{SO}_{2}$-Verteilung bei den oben dargestellten winterlichen Hochdrucklagen mit NW- bis NE-Höhenströmung dargestellt (Fig.6). Der rechte Teil der Figur zeigt eine typische $\mathrm{SO}_{2}$-Verteilung während einer entsprechenden Wetterperiode. Die Auswertung der zehn seit 1968 vorbildlich betreuten Stationen des Bieler Lebensmittelinspektorates läßt sich ausgezeichnet mit der auf Figur 5 dargestellten Durchlüftungskarte korrelieren: Die Zone höchster $\mathrm{SO}_{2}$-Konzentration zieht sich vom Stadtzentrum in Leewindrichtung (gültig für Gradient- und Talwindeinfluß!) gegen den See hinunter. Ein sekundäres Maximum ist im Bereich der häufigen Mischungsschichtobergrenze (Untergrenze der Höheninversion) zu lokalisieren!
Eine Erklärung für die Entstehung dieser beiden Maxima läßt sich anhand der linken Darstellung finden (Fig. 6): Die Kurzfristmessungen $\left(\mathrm{SO}_{2}\right.$-Detektoren Philips PW 9700) im Stadtzentrum und am Jurahang während einer entsprechenden Wetterlage zeigen, da $B$ sich im Raum Zentralplatz bei konstant hohen $\mathrm{SO}_{2}$-Werten sehr oft ein zweigipfliger Tagesgang einstellt (Verkehrsspitzen!). Beim Spital Vogelsang können bei großen Schwankungen vor allem am Mittag ausgesprochene Extremwerte registriert werden. Die klimatologische Analyse zeigt, da $\beta$ dieses Phänomen auf die in Kapitel 4.5 erwähnte und infolge der hohen Vertikalstabilität ausgelöste Abbremsung der täglichen Hangaufwinde zurückgeführt werden kann (siehe v.a. Kurvenverläufe des 9. und 12.12.1980). Obschon das Durchlüftungspotential (WANNER 1982) bei derartigen Höheninversionslagen größer ist als bei ausgesprochen schwachwindigen Bodeninversionssituationen, muß diese «Fumigation"-Lage infolge ihrer Persistenz im Hochwinter eindeutig als gefährlicher angesprochen werden!

\subsection{Eine erste Korrelation mit Atemwegserkrankungen bei Kindern}

Obwohl das $\mathrm{SO}_{2}$ eine lufthygienische Leitgröße darstellt, ist seine Korrelation mit Folgewirkungen nur bedingt zulässig, da bei Lebewesen in erster Linie die Wirkung des Gesamtkomplexes Wetter-/Lufthygiene betrachtet werden muß! Die Aufzeichnung von 132 Fällen von Atemwegserkrankungen bei Kindern (Winterhalbjahr 1980/81) zeigen eine eindeutige Häufung der Krankheitsfälle bei den oben dargestellten Hochdrucklagen mit nördlichen bis östlichen Höhenströmungen: $31 \%$ aller $\mathrm{K}$ rankheitsfälle bei einer relativen Wetterlagenhäufigkeit von nur 21\% (RICKLI 1982).

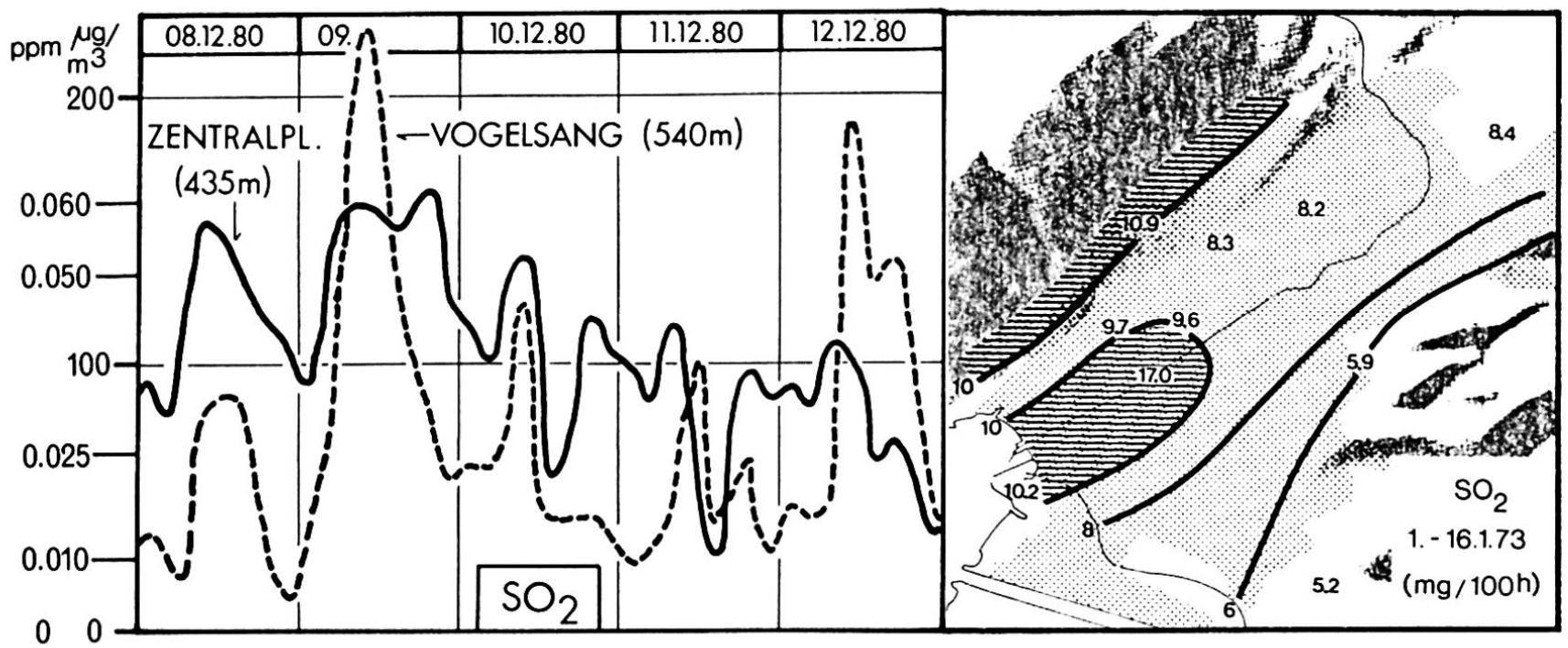

Fig. 6 Die $\mathrm{SO}_{2}$-Konzentrationen der Bieler Stadtluft bei winterlichen Hochdrucklagen mit nördlicher Höhenströmung (links: Kurzzeitmessungen; rechts: Mittelwerte über 15 Tage) 
Steht bei dieser Wetterlage eindeutig der Pseudocroup an der Spitze der aufgetretenen Erkrankungen, so ist bei feuchterem Milieu mit stärkeren Winden eine Verschiebung zur akuten Bronchitis hin feststellbar. Wenn auch die Zahl der Stichproben noch eindeutig zu gering ist, kann doch ein klarer Trend beobachtet werden!

Aus der erfreulicherweise sehr hohen Korrelation der langfristigen mit den kurzfristigen $\mathrm{SO}_{2}$-Messungen (geringer Einfluß chemischer Veränderungen auf die Langfristmessungen; BERLINCOURT 1981) lassen sich für die zwei Hauptimmissionszonen mit Hilfe einer einfach linearen Regression ganz grob die folgenden winterlichen 24-Std.-Höchstmittelwerte schätzen:

- Stadtzentrum: ca. $200 \mu \mathrm{g} / \mathrm{m}^{3}$

- Jurahang: ca. $120 \mu \mathrm{g} / \mathrm{m}^{3}$

Obschon diese Belastung als mäßig bezeichnet werden kann, ist eine Einwirkung auf empfindliche menschliche Organismen keineswegs ausgeschlossen (SEINFELD 1975)!

\subsection{Auswirkungen auf Planungsmaßnahmen}

Es ist höchst erfreulich, daß sich die Ergebnisse der Studie trotz anfänglicher Verständigungsschwierigkeiten in die laufenden Planungsentscheide umsetzen lassen. Folgende Probleme stehen zur Zeit im Mittelpunkt der Diskussionen mit den zuständigen Planungsstellen von Stadt und Region Biel (Stichworte):

- Autobahn N5/T6: Linienführung, Abluftkanäle, Untertunnelung

- Siedlungsplanung: Wohnsiedlungen am südlichen Seeufer oder am Jurahang (?); Beziehung zur Industriezone Bözingenmoos im NE der Stadt (Arbeitswege, Verkehrsemissionen?)

- Planung von Erholungsgebieten: Jurahang, Jurahöhen, See- und Flußufer, bewaldete Hügelgebiete

- Städtisches Energiekonzept: Emissionskataster, anthropogene Energie- und Wärmeproduktion, Abwärmenutzung

\section{Fortsetzung der Arbeiten}

In Zukunft werden die folgenden Arbeitsthemen im Vordergrund der Bieler Untersuchungen stehen (Stichworte; siehe Fig. 2):

- Emission: Umrechnung der einzelnen Emittentengruppen in den Gesamtraster (Hektare)

- Klima: Modellabschätzungen für Bodenbedeckung, Bodenrauhigkeit und Energieumsatz; Korrelation der Resultate mit episodischen Messungen dieser Größen bei typischen Wetterlagen

- Modelle: Ausbau und Anwendung der Modelle für raumplanerische Prognosen

- Immission: Ausweitung auf $\mathrm{NO}_{\mathrm{x}}$ und evtl. $\mathrm{O}_{3}$ (Einfluß Straßenverkehr!)
- Folgewirkungen: Erweiterung der Stichproben; Versuch einer geschickten Parametrisierung des Gesamtkomplexes Klima-Lufthygiene in Form von "Ausbreitungswetterlagen» (als Grundlage für eine Korrelation mit den Untersuchungen an Mensch und Pflanze)

- Planungsmaßnahmen: Autobahnlinienführung N5/T6, städtisches Energiekonzept

Grundsätzlich wird angestrebt, diese interdisziplinäre Studie mit ähnlichen Untersuchungen innerhalb der Schweiz zu vergleichen (u.a mit den anlaufenden Arbeiten im Rahmen des Nationalen Forschungsprogrammes «Lufthaushalt und Luftverschmutzung in der Schweiz»).

\section{Verdankungen}

Die Verfasser bedanken sich bei den folgenden Personen oder Institutionen für fachliche, personelle und finanzielle Unterstützung:

- Regionalplanungsverein Biel-Seeland

- Lufthygienefachstellen der Kantone Bern (H. Mathys), Aarau (E. Rüesch) und Solothurn (H. Rivar)

- Geographisches Institut der Universität Bern (Prof. B. Messerli)

- Colorado State University, Department of Atmospheric Science (Prof. E. R. Reiter, J. D. Sheaffer)

- Lebensmittelinspektorat der Stadt Biel (E.Bandi, J. Frank)

\section{Abstract}

An urban climate and air pollution study in the small town of Biel (Switzerland; population: 85000 including suburbs) aims not only at the analysis of a very dense distribution of emissions, dispersion climate and depositions, but also attempts to correlate these factors with selected effects on man. These effects include the impacts on children's respiratory organs and the growth of plants (lichen). The findings of this study will have direct application in land use planning and related political decisions for new highway systems, selection of new housing developments and industrial districts, as well as future fuel supply options.

Initial results show that future activities should concentrate on case studies during anticyclonic wintertime weather types with north-northeasterly synoptic flow. These weather types are associated with cold air advection, weak winds, strong temperature inversions and consequently, high air pollution rates in the Biel area. A first draft of a very dense analysis of the air pollution potential for a similar weather type has been worked out (figure 5). This analysis will be used for the design of additional mobile measurements and modeling studies. 


\section{Literatur}

AESCHBACHER, R. und MELI, R. (1977): Die SchwefeldioxydBelastung der Bieler Stadtluft. Hausarbeit Geogr. Inst. Univ. Bern, $56 \mathrm{~S}$.

BACH, W. (1970): An urban circulation model. In: Archiv Meteorol. Geoph. u. Bioklim., Ser. B, 18: 155-168.

BERLINCOURT, P. (1981): Immissionsmessungen im Winterhalbjahr 1980-1981. Zwischenber. z. d. klim.-lufthyg. Unters. im Raum Biel-Seeland, $36 \mathrm{~S}$.

EGLI, H.R. und WANNER, H. (1980): Klima und Wetter von Biel. In: Jahrbuch d. Geogr. Ges. v. Bern, Bd. 53: 1-25.

ERIKSEN, W. (1975): Probleme der Gelände- und Stadtklimatologie. Erträge der Forschung, Band 35, Wiss. Buchges. Darmstadt, 114 S.

FETT, W. (1974): Ein Index für das Stagnieren der bodennahen Luft. Beilage zur Berliner Wetterkarte, 14.3.1974, 12 S.

ITTEN, K. (1982): Beitrag zu einer Neu-Definition der Geographie. In: Geogr. Helvetica. Nr. 1, 35-37.

KLIMA UND PLANUNG 79 (1980): Tagungsbericht. Veröffentl. d. Geogr. Komm. d. Sz. Naturfor. Ges., Nr. 6, 193 S.

LETTAU, H.H. (1969): Note on aerodynamic roughness elementdescription. In: J. Appl. Meteorol., Nr. 8, 828-832.

MATHYS, H., MESSERLI, B., MAURER, R., WANNER, $H$. und WINIGER, M. (1980): Klima und Lufthygiene im Raum Bern. Veröffentl. d. Geogr. Komm. d. Sz. Naturfor. Ges., Nr. 7, 40 S.
OKE, T.R. (1978): Boundary layer climates. Methuen, London, 372 S.

OKE, T.R. (1981): Canyon geometry and the nocturnal urban heat island: comparison of scale model and field observations. In: Journ. of Climatology, Vol. 1, 237-254.

OKE, T.R. (1982): The energetic basis of the urban heat island. In: Quart. Journ. of the Roy. Meteorol. Soc., 108: 1-24.

RICKLI, R. (1982): Wind- und Temperaturfeld im Raum Biel im Winterhalbjahr 1980/81. Dipl. arbeit Geogr. Inst. Univ. Bern, $85 \mathrm{~S}$.

SEINFELD, J.H. (1975): Air Pollution. Physical and chemical fundamentals. McGraw-Hill, New York, 523 S.

SPERA, D.A. und RICHARDS, T.R. (1978): A revised wind shear power law model. Wind Energy Project Office, Project Inform. Release No. 70, NASA, $8 \mathrm{~S}$.

TAPPER, N.J., TYSON, P.D., OWENS, L.F. und HASTIE, W.J. (1981): Modeling the winter urban heat island over Christchurch, New Zealand. In: Journ. Appl. Meteorol., 20, 365-376.

WANNER, H. (1982): Eine Detailanalyse der Durchlüftung in der Schweiz - Konzept und erste Resultate. Tag'ber. 17. Int. Tag. f. Alpine Meteorol., Berchtesgaden. $3 \mathrm{~S}$.

WEISCHET, W. (1980): Stadtklimatologie und Stadtplanung. Klima und Planung 79. Veröffentl. d. Geogr. Komm. d. Sz. Naturfor. Ges., Nr. 6, 73-95. 